\title{
A Theorem for Finding Maximum Temperature in Wet Grinding
}

\author{
J. L. González-Santander and G. Martín \\ Departamento de Ciencias Experimentales y Matemáticas, Universidad Católica de Valencia "San Vicente Mártir", \\ C/Guillem de Castro 96, 46001 Valencia, Spain \\ Correspondence should be addressed to J. L. González-Santander; martinez.gonzalez@ucv.es
}

Received 10 February 2015; Revised 18 May 2015; Accepted 28 May 2015

Academic Editor: Bogdan Dumitrescu

Copyright (C) 2015 J. L. González-Santander and G. Martín. This is an open access article distributed under the Creative Commons Attribution License, which permits unrestricted use, distribution, and reproduction in any medium, provided the original work is properly cited.

\begin{abstract}
We consider the solutions found in the literature for heat transfer in surface grinding, assuming a constant heat transfer coefficient for the coolant acting on the workpiece surface and a constant or linear heat flux profiles entering into the workpiece. From the integral form of the time-dependent temperature field reached in the workpiece, assuming the previous conditions, we prove that the maximum temperature always occurs in the stationary regime on the workpiece surface within the contact zone between the wheel and the workpiece. This result assures a very rapid method for the theoretical computation of the maximum temperature.
\end{abstract}

\section{Introduction}

Surface grinding is an industrial machining process aiming to remove excess material of a workpiece by means of an abrasive wheel which rotates at high speed over its surface (see Figure 1). Most of the energy used in the grinding process is converted into heat, and it is accumulated within the contact zone between the wheel and the workpiece [1]. The high temperatures reached can thermally damage the quality of the workpiece, burning the workpiece, causing metallurgical phase transformations, softening (tempering) the surface layer with possible rehardening, onsetting residual tensile stresses, and causing cracks $[1,2]$. Therefore, the determination of the temperature field evolution inside the workpiece is of great industrial importance [3-7]. In order to avoid thermal damage, coolant is usually delivered to the porous grinding zone at a high velocity, so friction is reduced and cooling by convection occurs. Some recommendations for the optimization of the coolant usage are found in [8].

1.1. Heat Transfer Model in Surface Grinding. In surface grinding, the heat transfer inside the workpiece is usually modeled [9-11] by a strip heat source infinitely long and of $2 \ell$ width ( $\mathrm{m}$ in SI units), which moves at a speed $\vec{v}_{f}=v_{f} \vec{i}\left(\mathrm{~m} \mathrm{~s}^{-1}\right)$ over a semi-infinite solid surface (see Figure 1). Notice that, without losing generality, we can consider

$$
v_{f}>0,
$$

because reversing the motion of the workpiece relative to the grinding wheel, $v_{f} \rightarrow-v_{f}$, is equivalent to change the direction of the $x$-axis, $x \rightarrow-x$.

Setting the Cartesian coordinate system $X Y Z$ fixed to wheel, as shown in Figure 1, the temperature field of the workpiece $T(t, x, z)$ must satisfy the convective heat equation (notice that if $\vec{v}_{d}$ represents the velocity of the motion of the workpiece, then $\left.\vec{v}_{d}=-\vec{v}_{f}\right)[12, \$ 1.7(2)]$ :

$$
\frac{\partial T}{\partial t}=k\left(\frac{\partial^{2} T}{\partial x^{2}}+\frac{\partial^{2} T}{\partial z^{2}}\right)+v_{f} \frac{\partial T}{\partial x},
$$

where $k$ is the thermal diffusivity $\left(\mathrm{m}^{2} \mathrm{~s}^{-1}\right)$. Since initially the workpiece is at room temperature $T_{0}(\mathrm{~K}),(2)$ is subjected to the following initial condition:

$$
T(0, x, z)=T_{0} .
$$

From a theoretical point of view, heat transfer in wet surface grinding has been modelled assuming a constant 


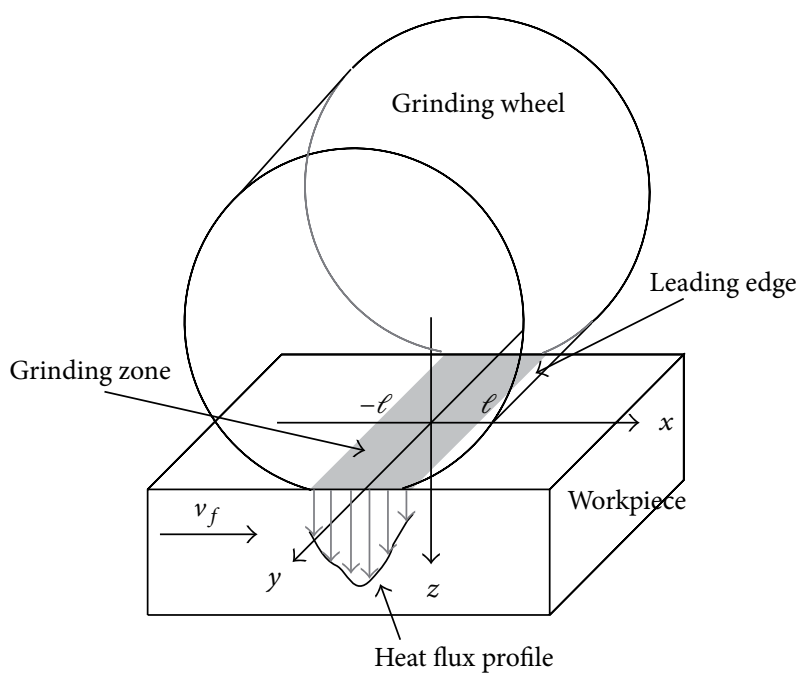

FIGURE 1: Setup in surface grinding.

heat transfer coefficient $h\left(\mathrm{~W} \mathrm{~m}^{-2} \mathrm{~K}^{-1}\right)$ on the workpiece surface $[11,13]$. Despite the fact that this is not a very realistic assumption, because outside and inside the grinding zone are expected to have different values of $h$ [3], it is known that most of the energy removed by convection due to coolant occurs within the grinding zone [14]. Therefore, considering that the actual $h$ inside the grinding zone is equal to the one outside of it (constant $h$ assumption), it is expected that the temperature field does not differ very much from a nonconstant $h$ assumption. Moreover, the assumption of constant $h$ makes the problem to be analytically tractable. Assuming as well a dimensionless heat flux profile $f(x)$ within the contact area between wheel and workpiece, we have the following boundary condition:

$$
\begin{aligned}
k_{0} \frac{\partial T}{\partial z}(t, x, 0)= & h\left[T(x, 0, t)-T_{0}\right] \\
& -q f(x) \theta(x-\ell) \theta(x+\ell),
\end{aligned}
$$

where $\theta(x)$ denotes the Heaviside function, $k_{0}\left(\mathrm{~W} \mathrm{~m}^{-1} \mathrm{~K}^{-1}\right)$ is the thermal conductivity, and $q\left(\mathrm{~W} \mathrm{~m}^{-2}\right)$ is the average heat flux entering into the workpiece along the contact width $2 \ell$; thus

$$
\frac{1}{2 \ell} \int_{-\ell}^{\ell} f(x) d x=1 .
$$

1.2. The Solutions. This paper will focus on the issue from a theoretical point of view by using the solution of (2)-(4) given by DesRuisseaux [10] for a constant heat flux profile and the solution given by Sauer [11] for a linear heat flux profile. In dry grinding, experiments suggest that the heat flux profile is linear (triangular with its apex at the inlet of the wheel-workpiece contact arc) in the case of upcutting [15]. Moreover, in wet grinding, according to [14], a constant heat flux profile is expected to occur in downcutting, while a linear heat flux profile is presumed to occur in upcutting. However, a linear heat flux profile may prevail in the literature, although a constant profile is used as well [3].
For both heat flux profiles, Green's method is used to arrive to the desired solution. Assuming a Cartesian coordinate system fixed to the wheel (see Figure 1), the Green function $G(t, x, y, z)$ is interpreted in this case as the temperature field rise with respect to the room temperature, evaluated at $(t, x, y, z)$ in a semi-infinite body $(z>0)$ in which, at the position over the surface $\left(x_{0}, y_{0}, 0\right)$, an instantaneous point heat source of energy $E(J)$ appears at the instant $t_{0}<t$, where the surface $(z=0)$ is subject to a constant heat transfer coefficient $h$ :

$$
\begin{aligned}
& G(t, x, y, z)=\frac{E}{4 \pi k_{0}\left(t-t_{0}\right)} \\
& \cdot \exp \left(-\frac{\left(x+v_{f}\left(t-t_{0}\right)-x_{0}\right)^{2}+\left(y-y_{0}\right)^{2}}{4 k\left(t-t_{0}\right)}\right) \\
& \cdot\left\{\frac{1}{2 \sqrt{\pi k\left(t-t_{0}\right)}} \exp \left(-\frac{z^{2}}{4 k\left(t-t_{0}\right)}\right)\right. \\
& -\widetilde{h} e^{\widetilde{h} z+\tilde{h}^{2} k\left(t-t_{0}\right)} \\
& \left.\cdot \operatorname{erfc}\left(\tilde{h} \sqrt{k\left(t-t_{0}\right)}+\frac{z}{2 \sqrt{k\left(t-t_{0}\right)}}\right)\right\}
\end{aligned}
$$

where the radiation coefficient $\left(\mathrm{m}^{-1}\right)$ is defined as

$$
\tilde{h}=\frac{h}{k_{0}} \text {. }
$$

A formal derivation of (6) is given in [16].

The derivation of the solution of (2)-(4) by using (6) follows three steps:

(i) Superposition in space to give temperature due to instantaneous line source, which acts on the surface $z=0$ parallel to the $y$-axis.

(ii) Superposition of line sources to give temperature due to a instantaneously acting strip source on the surface.

(iii) Superposition in time to give temperature due to continuously acting strip source.

Let us consider now on the following dimensionless variables, which, taking into account (1), satisfy

$$
\begin{aligned}
& X=\frac{v_{f} x}{2 k} \in \mathbb{R}, \\
& Z=\frac{v_{f} z}{2 k} \geq 0, \\
& L=\frac{v_{f} \ell}{2 k}>0, \\
& H=\frac{2 k h}{v_{f} k_{0}}>0,
\end{aligned}
$$




$$
\begin{aligned}
\tau & =\frac{v_{f} \sqrt{t}}{2 \sqrt{k}}>0, \\
\mathscr{T} & =\frac{\pi k_{0} v_{f}}{2 k q}\left(T-T_{0}\right)>0 .
\end{aligned}
$$

In the literature, $L$ is termed as the Peclet number and $H$ as the Biot number. For a constant heat flux profile satisfying (5),

$$
f^{(0)}(x)=1
$$

DesRuisseaux in [10] provides the following solution:

$$
\mathscr{T}^{(0)}(\tau, X, Z)=\int_{0}^{\tau} \rho(Z, w) F^{(0)}(X, w) d w,
$$

where

$$
\begin{aligned}
\rho(Z, w)= & \exp \left(\frac{-Z^{2}}{4 w^{2}}\right) \\
& -\sqrt{\pi} H w e^{H Z+H^{2} w^{2}} \operatorname{erfc}\left(H w+\frac{Z}{2 w}\right), \\
F^{(0)}(X, w)= & \sqrt{\pi}\left[\operatorname{erf}\left(\frac{u}{2 w}+w\right)\right]_{u=X-L}^{X+L} .
\end{aligned}
$$

Similarly, for a linear heat flux profile

$$
f^{(1)}(x)=1+\frac{x}{\ell}
$$

Sauer in [11] provides the following solution:

$$
\mathscr{T}^{(1)}(\tau, X, Z)=\int_{0}^{\tau} \rho(Z, w) F^{(1)}(X, w) d w
$$

where

$$
\begin{aligned}
& F^{(1)}(X, w)=\frac{1}{L}\left[\sqrt{\pi}\left(X+L+2 w^{2}\right) \operatorname{erf}\left(\frac{u}{2 w}+w\right)\right. \\
& \left.+2 w \exp \left(-\left[\frac{u}{2 w}+w\right]^{2}\right)\right]_{u=X-L}^{X+L} .
\end{aligned}
$$

It is worth noting that an equivalent solution to (14) can be found in [16] within the framework of the Samara-Valencia model [17].

Notation. We will drop the superscripts ${ }^{(0)}$ or ${ }^{(1)}$ when we refer to the temperature field $\mathscr{T}(\tau, X, Z)$ regardless of the heat flux profile entering into the workpiece, so that we will write simultaneously (10) and (14) as

$$
\mathscr{T}(\tau, X, Z)=\int_{0}^{\tau} \rho(Z, w) F(X, w) d w
$$

1.3. Maximum Temperature Search Approach. In order to avoid thermal damage, we need to control the maximum temperature $\mathscr{T}_{\text {max }}$ in grinding, calculated from the temperature fields expressions given in (10) and (14). Therefore, let us state the following theorem, which will be very useful for the numerical computation of $\mathscr{T}_{\text {max }}$.

Theorem 1. The maximum temperature $\mathscr{T}_{\max }$ of the timedependent temperature field $\mathscr{T}(\tau, X, Z)$ is

$$
\mathscr{T}_{\text {max }}=\lim _{\tau \rightarrow \infty} \mathscr{T}\left(\tau, X_{\max }, 0\right),
$$

where

$$
X_{\max } \in[-L, L]
$$

The proof of Theorem 1 follows three steps:

(1) The maximum temperature is reached in the stationary regime, that is to say, when $\tau \rightarrow \infty$.

(2) The maximum temperature is reached on the workpiece surface, that is to say, at $Z=0$.

(3) The maximum temperature is located within the grinding zone, that is to say, $X_{\max } \in[-L, L]$.

As far as author's knowledge, the occurrence of the maximum temperature in the stationary regime is taken for granted in the literature and we did not find any formal proof of it. Moreover, we have found the following considerations about the maximum temperature:

(i) Jaeger calculates in [9] the temperature field in the case of dry grinding, considering a constant heat flux profile. He takes for granted that the maximum temperature occurs in the stationary regime on the workpiece surface. Moreover, he provides some approximations for large $L$ in order to calculate the surface temperature in the stationary regime, from which he estimates the maximum temperature.

(ii) Malkin and Guo in [3] follow Jaeger approximated formula for maximum temperature.

(iii) DesRuisseaux in [13] presents the temperature field in wet grinding for a constant heat flux profile. He does not consider his solution in the transient regime. Also, he plots the temperature for $H=1$ and for different depths $Z$, in order to illustrate that the maximum temperature occurs on the surface and within the grinding zone.

(iv) Sauer in [11] calculates the temperature field in wet grinding for a linear heat flux profile, but again he considers his solution only in the stationary regime. He says that the region in which the maximum temperature is expected lies on

$$
\begin{aligned}
-2 & \leq \frac{x}{\ell} \leq 1, \\
0 & \leq \frac{z}{\ell} \leq 2 .
\end{aligned}
$$


Therefore, it might be expected that the maximum temperature occurs outside the grinding zone and at certain depth from the surface.

However, for the case of a constant heat flux profile, we find in the literature the following partial results regarding to Theorem 1:

(i) The maximum temperature occurs when $t \rightarrow \infty[18$, Sect. 3.1].

(ii) When $t \rightarrow \infty$, the temperature field reaches the stationary regime; that is, $\partial T / \partial t=0[16$, Sect. 5.2]

(iii) When the temperature field reaches the stationary regime, the maximum temperature occurs at the boundary, that is, on the workpiece surface $[18$, Sect. 3.2].

(iv) In order to locate the maximum temperature in the stationary regime on the workpiece surface, it can be proved for small Biot numbers that always this maximum occurs within the grinding zone $[18$, Sect. 3.3].

It is worth noting also that the numerical simulation of the time-dependent field temperature by using FEM analysis [19] agrees with Theorem 1.

The principal scope of this paper is just to generalize the above results for any Biot number, for both a constant and a linear heat flux profiles. We will see later on that this is a challenging mathematical problem.

This paper is organized as follows. Section 2 is devoted to prove that the maximum temperature is found on the surface in the stationary regime, for both a linear and a constant heat flux profiles. Section 3 proves that the maximum temperature occurs within the grinding zone, regardless as well of the heat flux profile considered: constant or linear. Section 4 provides a numerical example of the temperature field in the stationary regime, considering a constant and a linear heat flux profile. Our conclusions are summarized in Section 5. In the Appendix we collect some auxiliary lemmas in order to support the results given in body of the paper.

\section{Maximum Temperature on the Surface in the Stationary Regime}

In this section we will prove first that $\mathscr{T}(\tau, X, Z)$ is a monotonically increasing function of $\tau$ (Theorem 4 ), so that $\mathscr{T}_{\text {max }}$ must be asymptotically reached when $\tau \rightarrow \infty$. This result prevents the occurrence of a temperature peak during the transient regime. Once this is proved, we will prove that when $\tau \rightarrow \infty$, the temperature field does not evolve over time; that is, the stationary regime is asymptotically reached when $\tau \rightarrow \infty$ (Proposition 8). This fact is the key result to prove that the temperature field in the stationary regime can be interpreted as a harmonic function, so that, according to the maximum principle of harmonics functions, the maximum temperature is found to be on the boundary, that is, on the workpiece surface (Theorem 9).
Proposition 2. The function $\rho(Z, w)$ satisfies

$$
\rho(Z, w)>0, \quad \forall w>0, Z \geq 0 .
$$

Proof. According to [20, Sect. 2.2], we have

$$
e^{x^{2}} \operatorname{erfc}(x)=\frac{1}{\sqrt{\pi}}\left(\frac{1}{x}-e^{x^{2}} \int_{x}^{\infty} \frac{e^{-t^{2}}}{t^{2}} d t\right) .
$$

Since

$$
\int_{x}^{\infty} \frac{e^{-t^{2}}}{t^{2}} d t>0, \quad \forall x \in \mathbb{R}
$$

we may rewrite (21) as

$$
e^{-x^{2}}-\sqrt{\pi} x \operatorname{erfc}(x)>0, \quad \forall x>0 .
$$

Moreover, the following inequality is also satisfied:

$$
e^{-x^{2}}-\sqrt{\pi}(x-a) \operatorname{erfc}(x)>0, \quad \forall x>0, a \geq 0 .
$$

Taking $x=H w+Z / 2 w>0$ and $a=Z / 2 w \geq 0$ in (24), we have

$$
\begin{aligned}
\exp & \left(-\frac{Z^{2}}{4 w^{2}}-H^{2} w^{2}-H Z\right) \\
& -\sqrt{\pi} H w \operatorname{erfc}\left(H w+\frac{Z}{2 w}\right)>0,
\end{aligned}
$$

so, according to (11), it follows (20), as we wanted to prove.

Proposition 3. The functions $F(X, w)$ satisfies

$$
F(X, w)>0, \quad \forall w>0, X \in \mathbb{R} .
$$

Proof. According to the definition of $F^{(0)}(X, w)$ given in (12), we may rewrite this function as

$$
F^{(0)}(X, w)=\frac{1}{w} \int_{X-L}^{X+L} \exp \left(-\left[\frac{u}{2 w}+w\right]^{2}\right) d u>0,
$$

which is positive since $X-L<X+L, w>0$, and the integrand is positive within the integration interval. Similarly, let us rewrite $F^{(1)}(X, w)$, defined in (15), as

$$
\begin{aligned}
F^{(1)} & (X, w) \\
& =\frac{1}{w} \int_{X-L}^{X+L}\left(1+\frac{X-u}{L}\right) \exp \left(-\left[\frac{u}{2 w}+w\right]^{2}\right) d u \\
& >0,
\end{aligned}
$$

which is also positive because $X-L<X+L, w>0$, and the integrand is positive within the integration interval.

Theorem 4. The time-dependent temperature field is a monotonically increasing function of $\tau$ :

$$
\frac{\partial \mathscr{T}(\tau, X, Z)}{\partial \tau}>0, \quad \forall \tau>0, X \in \mathbb{R}, Z \geq 0 .
$$


Proof. Performing the derivative with respect to $\tau$ in (16) and taking into account (20) and (26), we have

$$
\frac{\partial \mathscr{T}(\tau, X, Z)}{\partial \tau}=\rho(Z, \tau) F(X, \tau)>0,
$$

$$
\forall \tau>0, X \in \mathbb{R}, Z \geq 0
$$

Corollary 5. The maximum temperature $\mathscr{T}_{\max }$ is asymptotically reached when $\tau \rightarrow \infty$.

Let us prove now that the stationary regime is asymptotically reached at $\tau \rightarrow \infty$. For this purpose, let us start proving the following propositions.

Proposition 6. The function $\rho(Z, \tau)$ satisfies

$$
\lim _{\tau \rightarrow \infty} \rho(Z, \tau)=0, \quad \forall Z \in \mathbb{R} .
$$

Proof. According to the definition of the $\rho(Z, \tau)$ given in (11), we have

$$
\lim _{\tau \rightarrow \infty} \rho(Z, \tau)=1-\sqrt{\pi} \lim _{\tau \rightarrow \infty} H \tau e^{H^{2} \tau^{2}} \operatorname{erfc}(H \tau),
$$

$\forall Z \in \mathbb{R}$.

Taking into account now the asymptotic expansion [21, Eq. 7.12.1]

$$
\sqrt{\pi} e^{x^{2}} \operatorname{erfc}(x) \approx \sum_{k=0}^{\infty} \frac{(-1)^{k}(2 k-1) ! !}{2^{k} x^{2 k+1}}, \quad x \longrightarrow \infty,
$$

up to first order, we have

$$
\sqrt{\pi} \lim _{\tau \rightarrow \infty} e^{H^{2} \tau^{2}} \operatorname{erfc}(H \tau)=\lim _{\tau \rightarrow \infty} \frac{1}{H \tau}, \quad \tau \longrightarrow \infty,
$$

so, substituting (34) in (32), we conclude (31), as we wanted to prove.

Proposition 7. The functions $F(X, \tau)$ satisfy

$$
\lim _{\tau \rightarrow \infty} F(X, \tau)=0, \quad \forall X \in \mathbb{R} .
$$

Proof. According to the integral representation (27), we have

$$
\begin{aligned}
& \lim _{\tau \rightarrow \infty} F^{(0)}(X, \tau) \\
& =\lim _{\tau \rightarrow \infty} \frac{1}{\tau} \int_{X-L}^{X+L} \exp \left(-\left[\frac{u}{2 \tau}+\tau\right]^{2}\right) d u=0,
\end{aligned}
$$

since the integral given in (36) is finite for all $X \in \mathbb{R}$. Similarly, from (28), we have

$$
\begin{aligned}
& \lim _{\tau \rightarrow \infty} F^{(1)}(X, \tau) \\
& =\lim _{\tau \rightarrow \infty} \frac{1}{\tau} \int_{X-L}^{X+L}\left(1+\frac{X-u}{L}\right) \exp \left(-\left[\frac{u}{2 \tau}+\tau\right]^{2}\right) d u \\
& =0 .
\end{aligned}
$$

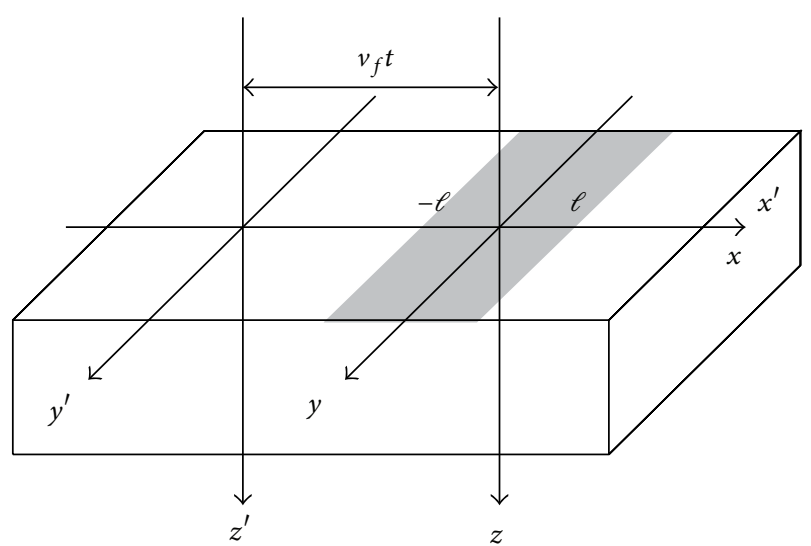

FIGURE 2: Relationship between the coordinates systems, one fixed to the wheel and the other one to the workpiece.

Proposition 8. The stationary regime is asymptotically reached, when $\tau \rightarrow \infty$ :

$$
\lim _{\tau \rightarrow \infty} \frac{\partial \mathscr{T}(\tau, X, Z)}{\partial \tau}=0, \quad \forall X, Z \in \mathbb{R}
$$

Proof. See that, from (16), we have

$$
\lim _{\tau \rightarrow \infty} \frac{\partial \mathscr{T}(\tau, X, Z)}{\partial \tau}=\lim _{\tau \rightarrow \infty} \rho(Z, \tau) F(X, \tau),
$$

and, according to (31) and (35), we conclude (38).

The above proposition allows us to prove now the following result.

Theorem 9. The maximum temperature $\mathscr{T}_{\max }$ is reached at the surface of the workpiece, $Z=0$.

Proof. Let us denote the Cartesian coordinates fixed to the workpiece as $X^{\prime} Y^{\prime} Z^{\prime}$ (see Figure 2), where, at $t=0$, both coordinates systems are overlapped. Consider as well the temperature field referred to $X^{\prime} Y^{\prime} Z^{\prime}$ as $T_{w}\left(t, x^{\prime}, z\right)$, where

$$
\begin{aligned}
& x^{\prime}=x+v_{f} t, \\
& z^{\prime}=z \\
& t^{\prime}=t
\end{aligned}
$$

Applying chain's rule, it is easy to see that the convective term in (2) disappears:

$$
\frac{\partial T_{w}}{\partial t}=k\left(\frac{\partial^{2} T_{w}}{\partial x^{\prime 2}}+\frac{\partial^{2} T_{w}}{\partial z^{2}}\right)
$$


Taking now similar dimensionless variables as in (8), that is to say,

$$
\begin{aligned}
X^{\prime} & =\frac{v_{f} x^{\prime}}{2 k}, \\
Z & =\frac{v_{f} z}{2 k}, \\
\tau & =\frac{v_{f} \sqrt{t}}{2 \sqrt{k}}, \\
\mathscr{T}_{w} & =\frac{\pi k_{0} v_{f}}{2 k q}\left(T_{w}-T_{0}\right),
\end{aligned}
$$

(2) becomes

$$
\frac{1}{2 \tau} \frac{\partial \mathscr{T}_{w}}{\partial \tau}=\frac{\partial^{2} \mathscr{T}_{w}}{\partial X^{\prime 2}}+\frac{\partial^{2} \mathscr{T}_{w}}{\partial Z^{2}} .
$$

According to (40) and (42), it is clear that the relationship between the temperature field referred to the workpiece $\mathscr{T}_{w}$ and the one referred to the wheel $\mathscr{T}$ is given by

$$
\mathscr{T}_{w}\left(\tau, X^{\prime}, Z\right)=\mathscr{T}\left(\tau, X+2 \tau^{2}, Z\right) .
$$

Since (38) is satisfied for all $X \in \mathbb{R}$, according to (44), we obtain

$$
\begin{aligned}
\lim _{\tau \rightarrow \infty} \frac{\partial \mathscr{T}_{w}\left(\tau, X^{\prime}, Z\right)}{\partial \tau} & =\lim _{\tau \rightarrow \infty} \frac{\partial \mathscr{T}\left(\tau, X+2 \tau^{2}, Z\right)}{\partial \tau} \\
& =0 .
\end{aligned}
$$

Thus, performing the limit $\tau \rightarrow \infty$ in (43), taking into account (45), we have

$$
\lim _{\tau \rightarrow \infty}\left(\frac{\partial^{2} \mathscr{T}_{w}}{\partial X^{\prime 2}}+\frac{\partial^{2} \mathscr{T}_{w}}{\partial Z^{2}}\right)=0
$$

so

$$
\lim _{\tau \rightarrow \infty} \mathscr{T}_{w}\left(\tau, X^{\prime}, Z\right)
$$

is a harmonic function. Therefore, according to the maximum principle of harmonics functions [22, Chap. VI], the maximum temperature of (47) occurs on the boundary, that is, on the workpiece surface, $Z=0$, as we wanted to prove.

\section{Maximum Temperature within the Grinding Zone}

According to Corollary 5 and Theorem 9, the maximum temperature $\mathscr{T}_{\max }$ must be found on the surface of the workpiece in the stationary regime, $\tau \rightarrow \infty$. Therefore, let us define the $\mathscr{T}_{s}(X)$ function as the surface temperature in the stationary regime:

$$
\begin{aligned}
\mathscr{T}_{s}(X) & =\lim _{\tau \rightarrow \infty} \mathscr{T}(\tau, X, 0) \\
& =\int_{0}^{\infty} \rho(0, w) F(X, w) d w .
\end{aligned}
$$

The idea is to prove that $\mathscr{T}_{s}(X)$ is a monotonically increasing (Theorem 14) or decreasing (Theorem 10) function outside the grinding zone. Therefore, $\mathscr{T}_{\max }$ must be found within the grinding zone, $X \in[-L, L]$, as it is stated in Theorem 1.

Theorem 10. The surface temperature in the stationary regime is a monotonically decreasing function on the right-hand side of the leading edge:

$$
\frac{d \mathscr{T}_{s}(X)}{d X}<0, \quad \forall X>L .
$$

Proof. From (48), we have

$$
\frac{d \mathscr{T}_{s}(X)}{d X}=\int_{0}^{\infty} \rho(0, w) \frac{\partial F(X, w)}{\partial X} d w
$$

where, according to (12),

$$
\frac{\partial F^{(0)}(X, w)}{\partial X}=\frac{1}{w}\left[\exp \left(-\left[\frac{u}{2 w}+w\right]^{2}\right)\right]_{u=X-L}^{X+L},
$$

and, according to (15),

$$
\begin{aligned}
L \frac{\partial F^{(1)}(X, w)}{\partial X}= & \sqrt{\pi}\left[\operatorname{erf}\left(\frac{u}{2 w}+w\right)\right]_{u=X-L}^{X+L} \\
& -\frac{2 L}{w} \exp \left(-\left[\frac{X-L}{2 w}+w\right]^{2}\right) .
\end{aligned}
$$

Let us set now the following variables:

$$
\begin{aligned}
& a=\frac{X+L}{2 w}+w, \\
& b=\frac{X-L}{2 w}+w .
\end{aligned}
$$

thus

$$
a-b=\frac{L}{w}>0,
$$

since the integration interval in (50) means that $w>0$. Notice also that $X>L$ implies that $b>0$; so taking into account (54), we have

$$
a>b>0 .
$$

On the one hand, rewriting now (51) in terms of $a$ and $b$, we have

$$
\frac{\partial F^{(0)}(X, w)}{\partial X}=\frac{e^{-a^{2}}-e^{-b^{2}}}{w}<0, \quad \forall w>0, X>L,
$$

due to (55) and the fact that the $e^{-x^{2}}$ function is a monotonically decreasing function for positive arguments [23, Sect. 1.3. Def. 1].

On the other hand, (52) in terms of $a$ and $b$ reads out as

$$
\begin{aligned}
L \frac{\partial F^{(1)}(X, w)}{\partial X}= & \sqrt{\pi}[\operatorname{erf}(a)-\operatorname{erf}(b)] \\
& -2(a-b) e^{-b^{2}}
\end{aligned}
$$


Applying the mean value theorem for derivatives [24, Theorem 4.5], we have

$$
\frac{\operatorname{erf}(a)-\operatorname{erf}(b)}{a-b}=\operatorname{erf}^{\prime}(c)=\frac{2}{\sqrt{\pi}} e^{-c^{2}}, \quad c \in(a, b) .
$$

Therefore, $a>c>b>0$, so $e^{-c^{2}}<e^{-b^{2}}$ and (58) is written as

$$
\frac{\operatorname{erf}(a)-\operatorname{erf}(b)}{a-b}<\frac{2}{\sqrt{\pi}} e^{-b^{2}} .
$$

Taking into account (59) in (57), we conclude

$$
\frac{\partial F^{(1)}(X, w)}{\partial X}<0, \quad \forall w>0, X>L .
$$

Finally, collecting the results (56) and (60) and taking into account (20), we may write

$$
\rho(0, w) \frac{\partial F(X, w)}{\partial X}<0, \quad \forall w>0, X>L,
$$

so integrating (61) over the interval $w \in(0, \infty)$, according to [25, Theorem 3.3.4], then (49) follows directly, as we wanted to prove.

In the above theorem we have just seen that the sign of $\mathscr{T}_{s}^{\prime}(X)$ for $X>L$ is determined by the sign of $\partial F(X, w) / \partial X$. This is not the case for $X<-L$; so before arriving to Theorem 14, we have to prove the following three propositions.

Proposition 11. Let

$$
\varrho(w)=\frac{\rho(0, w)}{w}
$$

Then $\varrho(w)$ is a positive and monotonically decreasing function for positive arguments:

$$
\begin{gathered}
\varrho(w)>0, \quad \forall w>0 \\
\varrho^{\prime}(w)<0, \quad \forall w>0 .
\end{gathered}
$$

Proof. Particularizing (20) to $Z=0$, we have

$$
\rho(0, w)>0, \quad \forall w>0,
$$

so it directly follows (63), since $w>0$.

In order to prove (64), rewrite $\varrho(w)$ as follows, taking into account (11):

$$
\varrho(w)=\sqrt{\pi} H\left[\frac{1}{\sqrt{\pi} H w}-e^{H^{2} w^{2}} \operatorname{erfc}(H w)\right] .
$$

Define now the function

$$
R(x)=\frac{1}{\sqrt{\pi} x}-e^{x^{2}} \operatorname{erfc}(x),
$$

so

$$
R^{\prime}(x)=\frac{2}{\sqrt{\pi}}-\frac{1}{\sqrt{\pi} x^{2}}-2 x e^{x^{2}} \operatorname{erfc}(x)
$$

Considering that $[20$, Sect. 2.2]

$$
e^{x^{2}} \operatorname{erfc}(x)=\frac{1}{\sqrt{\pi}}\left(\frac{1}{x}-\frac{1}{2 x^{3}}+\frac{3 e^{x^{2}}}{2} \int_{x}^{\infty} \frac{e^{-t^{2}}}{t^{4}} d t\right),
$$

we can say

$$
R^{\prime}(x)<0, \quad \forall x>0 .
$$

Since

$$
\begin{aligned}
\varrho(w) & =\sqrt{\pi} H R(H w), \\
\varrho^{\prime}(w) & =\sqrt{\pi} H^{2} R^{\prime}(H w),
\end{aligned}
$$

according to (70), it follows (64), as we wanted to prove.

Proposition 12. Let

$$
G(u, w)=\exp \left(-\left[\frac{u}{2 w}+w\right]^{2}\right)
$$

$$
\forall w \in(0, \infty), \forall u<0 .
$$

If

$$
u_{2}<u_{1}<0
$$

then consider the following.

(1) There is an unique crossing point $\bar{w}$ such that

$$
G\left(u_{1}, \bar{w}\right)=G\left(u_{2}, \bar{w}\right) .
$$

(2) The following inequalities are satisfied:

$$
\begin{aligned}
& G\left(u_{1}, w\right)>G\left(u_{2}, w\right), \quad w \in(0, \bar{w}), \\
& G\left(u_{1}, w\right)<G\left(u_{2}, w\right), \quad w \in(\bar{w}, \infty) .
\end{aligned}
$$

Proof. Notice that

$$
\lim _{w \rightarrow 0} G(u, w)=\lim _{w \rightarrow \infty} G(u, w)=0, \quad \forall u \in \mathbb{R},
$$

so the values $\bar{w} \rightarrow 0$ or $\infty$ satisfy (74). Nevertheless, these values do not belong to the dominion of $G(u, w)$. Therefore, let us substitute (72) in (74), that is to say,

$$
\exp \left(-\left[\frac{u_{1}}{2 \bar{w}}+\bar{w}\right]^{2}\right)=\exp \left(-\left[\frac{u_{2}}{2 \bar{w}}+\bar{w}\right]^{2}\right)
$$

so

$$
\frac{u_{1}}{2 \bar{w}}+\bar{w}= \pm\left(\frac{u_{2}}{2 \bar{w}}+\bar{w}\right) .
$$

The equation with the "+" sign leads to $u_{1}=u_{2}$ but this contradicts (73). The equation with the “-” sign leads to

$$
\bar{w}\left(u_{1}, u_{2}\right)=\frac{\sqrt{-\left(u_{1}+u_{2}\right)}}{2}>0,
$$


which is positive, according to (73), so it falls within the dominion of $G(u, w)$.

Notice that (79) is an actual crossing point between the graphs of $G\left(u_{1}, w\right)$ and $G\left(u_{2}, w\right)$ as functions of $w$ since

$$
\frac{\partial G\left(u_{1}, \bar{w}\right)}{\partial w} \neq \frac{\partial G\left(u_{2}, \bar{w}\right)}{\partial w} .
$$

Indeed

$$
\begin{aligned}
& \frac{\partial G(u, w)}{\partial w} \\
& \quad=\exp \left(-\left[\frac{u}{2 w}+w\right]^{2}\right)\left(\frac{u}{2 w}+w\right)\left(\frac{u}{w^{2}}-2\right),
\end{aligned}
$$

so, if

$$
\frac{\partial G\left(u_{1}, \bar{w}\right)}{\partial w}=\frac{\partial G\left(u_{1}, \bar{w}\right)}{\partial w}
$$

then

$$
u_{1}=u_{2}
$$

but this contradicts (73). Therefore, there is a unique crossing point $\bar{w} \in(0, \infty)$ satisfying $(74)$, as we wanted to prove.

In order to prove (75), see that the function $f(w)=$ $G(u, w)$ for given $u<0$ satisfies the assumptions of Lemma A.1 of the appendix:

(1) According to (72), $f(w)>0$, for all $w \in(0, \infty)$.

(2) According to (76), $\lim _{w \rightarrow 0} f(w)=\lim _{w \rightarrow \infty} f(w)=0$.

(3) Also, $\exists ! w_{\max } \in(0, \infty)$ such that $f^{\prime}\left(w_{\max }\right)=0$. Indeed, it is easy to prove that the only value $w_{\max } \in$ $(0, \infty)$ for $u<0$ satisfying

$$
f^{\prime}\left(w_{\max }\right)=0 \text {, }
$$

is given by

$$
w_{\max }(u)=\sqrt{\frac{-u}{2}}>0 .
$$

Then, according to (A.4), $w_{\max }(u)$ is the location of the absolute maximum of $G(u, w)$ for given $u<0$. Moreover, as it is shown in Figure 3, the crossing point $\bar{w}$ satisfies

$$
0<w_{\max }\left(u_{1}\right)<\bar{w}\left(u_{1}, u_{2}\right)<w_{\max }\left(u_{2}\right) .
$$

Indeed, taking into account (79) and (85) in (86), we arrive at

$$
u_{1}>\frac{u_{1}+u_{2}}{2}>u_{2}>0
$$

which is trivially satisfied and is coherent with (73).

Notice as well that substituting (85) in (72), we obtain

$$
G\left(u, w_{\max }(u)\right)=1, \quad \forall u<0 .
$$

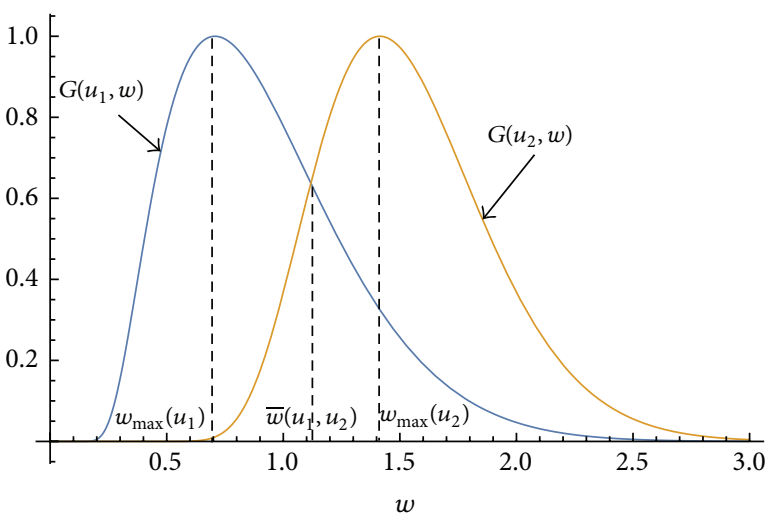

FIgURE 3: The crossing point $\bar{w}\left(u_{1}, u_{2}\right)$ lies between the $w_{\max }\left(u_{1}\right)$ and $w_{\max }\left(u_{2}\right)$.

Now, since $w_{\max }\left(u_{1}\right) \neq w_{\max }\left(u_{2}\right)$, by definition of absolute maximum, we have

$$
G\left(u_{2}, w_{\max }\left(u_{1}\right)\right)<G\left(u_{2}, w_{\max }\left(u_{2}\right)\right)=1,
$$

and taking into account (88), we obtain

$$
G\left(u_{2}, w_{\max }\left(u_{1}\right)\right)<G\left(u_{1}, w_{\max }\left(u_{1}\right)\right)=1 .
$$

See now that the functions $f_{1}(w)=G\left(u_{1}, w\right)$ and $f_{2}(w)=$ $G\left(u_{2}, w\right)$, where $u_{2}<u_{1}<0$, satisfy the assumptions of Lemma A.2 of the Appendix, that is to say,

(1) $\exists ! \bar{w} \in(a, b)$ such that $f_{1}(\bar{w})=f_{2}(\bar{w})$, according to (74);

(2) $f_{1}^{\prime}(\bar{w}) \neq f_{2}^{\prime}(\bar{w})$, according to $(80)$;

(3) taking $w_{0}=w_{\max }\left(u_{1}\right)$, according to (86) $w_{0} \in(0, \bar{w})$, and $(90)$ reads as $f_{1}\left(w_{0}\right)>f_{2}\left(w_{0}\right)$.

Therefore, the conclusion of Lemma A.2 given in (A.9)(A.10) is equivalent to (75), as we wanted to prove.

Proposition 13. The following improper integral is independent of $u$, if $u<0$ :

$$
\int_{0}^{\infty} G(u, w) d w=\frac{\sqrt{\pi}}{2}, \quad \forall u<0 .
$$

Proof. Using the definition (72) of the $G(u, w)$ function, we have

$$
\int_{0}^{\infty} G(u, w) d w=e^{-u} \int_{0}^{\infty} \exp \left(-\frac{u^{2}}{4 w^{2}}-w^{2}\right) d w .
$$

Applying the following integral [26, Eq. 3.325]

$$
\int_{0}^{\infty} \exp \left(-a x^{2}-\frac{b}{x^{2}}\right) d x=\frac{1}{2} \sqrt{\frac{\pi}{a}} \exp (-2 \sqrt{a b}),
$$

$$
a, b>0 \text {, }
$$

we arrive at

$$
\int_{0}^{\infty} G(u, w) d w=\frac{\sqrt{\pi}}{2} \exp (-u-|u|)
$$

so it follows directly (91), as we wanted to prove. 
Theorem 14. The surface temperature in the stationary regime is a monotonically increasing function on the left-hand side of the trailing edge:

$$
\frac{d \mathscr{T}_{s}(X)}{d X}>0, \quad \forall X<-L .
$$

Proof. Substituting in (58) the values of $a$ and $b$ given in (53) and taking into account (54), we have

$$
\sqrt{\pi}\left[\operatorname{erf}\left(\frac{u}{2 w}+w\right)\right]_{u=X-L}^{X+L}=\frac{2 L}{w} e^{-c^{2}}
$$

where

$$
\frac{X-L}{2 w}+w<c<\frac{X+L}{2 w}+w
$$

Taking into account that $X<-L$, rewrite $c$ as

$$
c=\frac{\xi}{2 w}+w, \quad X-L<\xi<X+L<0,
$$

so (97) is satisfied and (96) reads out as

$$
\begin{aligned}
\sqrt{\pi} & {\left[\operatorname{erf}\left(\frac{u}{2 w}+w\right)\right]_{u=X-L}^{X+L} } \\
& =\frac{2 L}{w} \exp \left(-\left[\frac{\xi}{2 w}+w\right]^{2}\right) .
\end{aligned}
$$

Substituting (99) in (52), we have

$$
\frac{\partial F^{(1)}(X, w)}{\partial X}=\frac{2}{w}\left[\exp \left(-\left[\frac{u}{2 w}+w\right]^{2}\right)\right]_{u=X-L}^{\xi} .
$$

Taking into account (51) and (100) and recalling the definitions of the $\varrho(w)$ and $G(u, w)$ functions, (72) and (62), respectively, let us express (50) as follows:

$$
\begin{aligned}
& \frac{d \mathscr{T}_{s}(X)}{d X} \\
& \quad=C \int_{0}^{\infty} \varrho(w)[G(u, w)-G(X-L, w)] d w,
\end{aligned}
$$

where $C=1$ or 2 and

$$
u=\left\{\begin{array}{l}
X+L \\
\xi
\end{array}\right.
$$

depending on whether we are in the constant or linear case, respectively. Therefore, according to (98) and (102), we have

$$
X-L<u \leq X+L<0 .
$$

Notice that the integral given in (101) satisfies the assumptions of Lemma A.3 given in the Appendix:

(1) According to Proposition 13 and taking into account (103), we have

$$
\int_{0}^{\infty} G(u, w) d w=\int_{0}^{\infty} G(X-L, w) d w .
$$

(2) According to Proposition 11, we have

$$
\begin{aligned}
\varrho(w)>0, & \forall w>0, \\
\varrho^{\prime}(w)<0, & \forall w>0 .
\end{aligned}
$$

(3) According to Proposition 12 and taking into account (103), we have

$$
\begin{array}{ll}
G(u, w)>G(X-L, w), & w \in(0, \bar{w}), \\
G(u, w)<G(X-L, w), & w \in(\bar{w}, \infty) .
\end{array}
$$

Therefore, since $C>0$, we conclude (95), as we wanted to prove.

\section{Numerical Example}

For the numerical evaluation of the temperature field, we will use the thermal properties of a VT20 titanium alloy workpiece [27]:

$$
\begin{aligned}
k_{0} & =13 \mathrm{~W} \mathrm{~m}^{-1} \mathrm{~K}^{-1}, \\
k & =4.23 \times 10^{-6} \mathrm{~m}^{2} \mathrm{~s}^{-1} .
\end{aligned}
$$

Also, we will take the following values for the grinding regime:

$$
\begin{aligned}
v_{f} & =0.53 \mathrm{~m} \mathrm{~s}^{-1}, \\
2 \ell & =2.663 \times 10^{-3} \mathrm{~m}, \\
q & =5.89 \times 10^{7} \mathrm{~W} \mathrm{~m}^{-2}, \\
h & =27.29 \times 10^{4} \mathrm{~W} \mathrm{~m}^{-2} \mathrm{~K}^{-1}, \\
T_{0} & =300 \mathrm{~K} .
\end{aligned}
$$

Figures 4 and 5 show the temperature field in the stationary regime $\lim _{t \rightarrow \infty} T(t, x, z)$ for a constant and a linear heat flux profile, respectively. Since the temperature gradient nearby the grinding zone is very high, note that the $z$-axis is zoomed with respect to the $x$-axis in order to obtain a more detailed view of the contour plots. Notice as well that, although the temperature fields are quite different in both graphs, the maximum temperature is found to be on the surface within the grinding zone. For a constant heat flux profile, the value and location on the surface of the maximum temperature is

$$
\begin{aligned}
& T_{\max }^{(0)} \approx 477.6 \mathrm{~K}, \\
& x_{\max }^{(0)} \approx-0.9727 \ell,
\end{aligned}
$$

and for a linear heat flux profile is

$$
\begin{aligned}
& T_{\max }^{(1)} \approx 537.3 \mathrm{~K}, \\
& x_{\max }^{(1)} \approx 0.6091 \ell .
\end{aligned}
$$




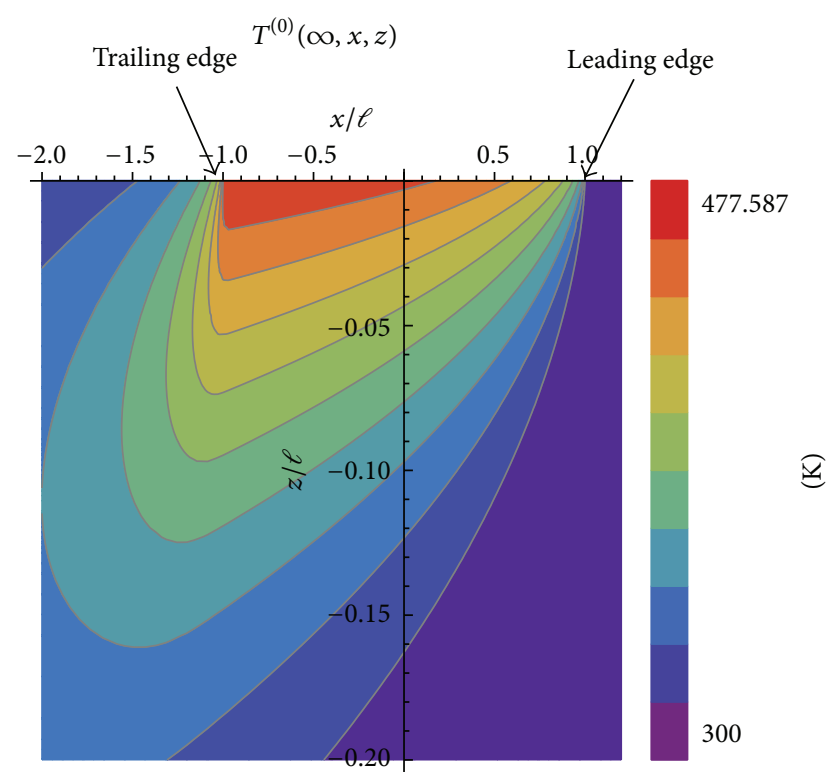

FIgURE 4: Temperature field for a constant heat flux profile in the stationary regime.

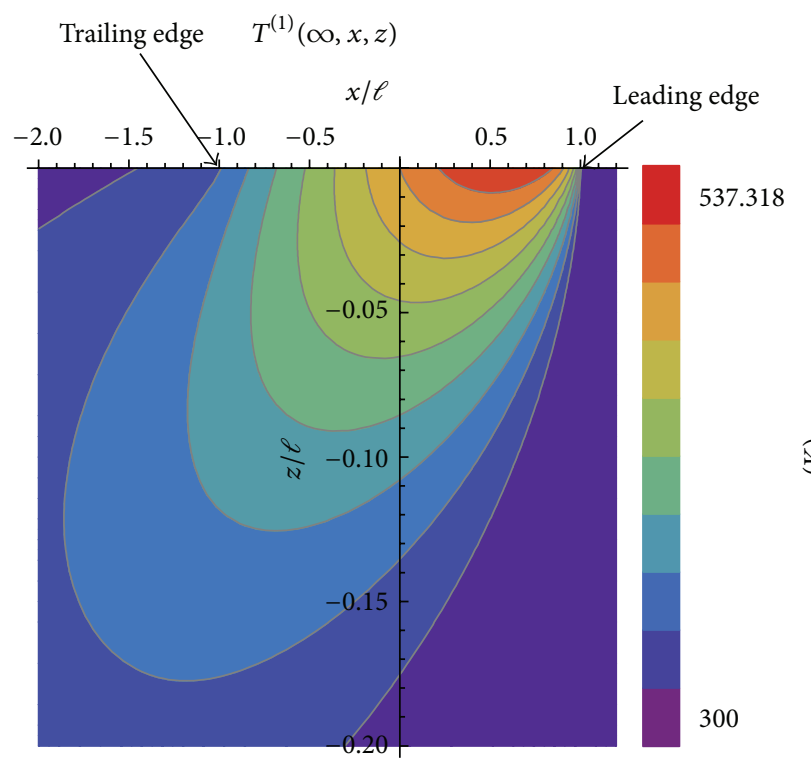

Figure 5: Temperature field for a linear heat flux profile in the stationary regime.

Note that the maximum temperature is higher in the linear case because the heat flux is skewed to the leading edge, but in the constant case the heat flux is uniform along the grinding zone. Nonetheless, the temperature field is more skewed to the trailing edge in the constant case because of the motion of the workpiece, being compensated the effect of the motion of the workpiece with the skewness of the heat flux profile in the linear case.

It is worth noting that the principal result of this paper (Theorem 1) assures a very rapid method for searching the maximum temperature. Indeed, the numerical search of the maximum in a three-dimensional field $\mathscr{T}(\tau, X, Z)$ within a unbounded region is reduced to the numerical computation of the maximum in a one-dimensional function $\mathscr{T}_{s}(X)$ within a particular interval; that is, $X_{\max } \in[-L, L]$.

\section{Conclusions}

We have considered the solutions found in the literature to model the heat transfer in surface grinding, assuming a constant and a linear heat flux profile entering into the workpiece and a constant heat transfer coefficient for the coolant acting on the workpiece surface. From these time-dependent temperature fields in dimensionless variables $\mathscr{T}(\tau, X, Z)$, we have analyzed the location of the maximum temperature in the workpiece, proving that the latter must be located on the workpiece surface, in the stationary regime and within the grinding zone, that is, Theorem 1 . As long as the heat transfer model stated in Section 1.1 can be applied to the real grinding process, Theorem 1 has the following consequences:

(i) The proof that the maximum temperature is found in the stationary regime prevents to encounter a temperature peak during the transient regime.

(ii) Also, the proof about the location of the maximum temperature on the workpiece surface prevents to find an internal thermal damage in the workpiece when its surface seems not to be damaged.

(iii) Moreover, the proof that the maximum temperature is located in the grinding zone on the surface and in the stationary regime converts the search of the maximum temperature in a three-dimensional temperature field within an unbounded region into the search of the maximum for a one-dimensional function within a particular interval, which is a much more simple problem from a computational point of view.

These theoretical results are intended to be useful in order to avoid thermal damage in surface wet grinding.

\section{Appendix}

\section{Auxiliary Lemmas}

Lemma A.1. Let $f:[a, b] \subseteq \overline{\mathbb{R}} \rightarrow \mathbb{R}$ be differentiable in $(a, b)$ and continuous in $[a, b]$ that satisfies

$$
\begin{aligned}
& f(a)=f(b)=0, \\
& f(x)>0, \quad x \in(a, b) .
\end{aligned}
$$

If

$$
\exists ! c \in(a, b) \text { such that } f^{\prime}(c)=0,
$$

then $c$ is an absolute maximum:

$$
f(c)=\max \{f(x), x \in[a, b]\} .
$$


Proof. Since the function $f(x)$ is continuous in $[a, b]$, according to the Extreme-Value Theorem of Continuous Functions [24, Theorem 3.12], the absolute maximum belongs to the interval $[a, b]$. Suppose that this absolute maximum is not located in $x=c$, say in $x=c^{\prime} \neq c$. Thus, by definition of absolute maximum and according to (A.2), we have

$$
f\left(c^{\prime}\right)>f(c)>0 .
$$

If $c^{\prime}=a$ or $b$, then, according to (A.1), $f\left(c^{\prime}\right)=0$, but this contradicts (A.5). If $c^{\prime} \in(a, b)$, then, since $f(x)$ is differentiable in $(a, b), c^{\prime}$ is also a local maximum, so $f^{\prime}\left(c^{\prime}\right)=0$, but this contradicts (A.3). Therefore, the absolute maximum must be located in $x=c$, as we wanted to prove in (A.4).

Lemma A.2. Let $f_{1}, f_{2}:(a, b) \rightarrow \mathbb{R}$ be two continuous and differentiable functions which have a single crossing point, that is to say,

$$
\begin{aligned}
\exists ! \bar{w} & \in(a, b) \quad \text { such that } f_{1}(\bar{w})=f_{2}(\bar{w}), \\
f_{1}^{\prime}(\bar{w}) & \neq f_{2}^{\prime}(\bar{w}) .
\end{aligned}
$$

If

$$
\exists w_{0} \in(a, \bar{w}) \quad \text { such that } f_{1}\left(w_{0}\right)>f_{2}\left(w_{0}\right),
$$

then

$$
\begin{aligned}
& f_{1}(w)>f_{2}(w), \quad \forall w \in(a, \bar{w}), \\
& f_{1}(w)<f_{2}(w), \quad \forall w \in(\bar{w}, b) .
\end{aligned}
$$

Proof. Let us consider the following differentiable function:

$$
g(w)=f_{1}(w)-f_{2}(w) .
$$

According to (A.6), we have

$$
g(\bar{w})=0 .
$$

Since $g$ is a differentiable function, according to (A.7), we have

$$
\begin{aligned}
& \lim _{h \rightarrow 0^{+}} \frac{g(\bar{w}+h)-g(\bar{w})}{h} \\
& =\lim _{h \rightarrow 0^{+}} \frac{g(\bar{w})-g(\bar{w}-h)}{h}=g^{\prime}(\bar{w}) \neq 0,
\end{aligned}
$$

so, according to (A.12),

$$
\lim _{h \rightarrow 0^{+}} \frac{g(\bar{w}+h)}{h}=\lim _{h \rightarrow 0^{+}} \frac{-g(\bar{w}-h)}{h} \neq 0 .
$$

Therefore, $g$ changes sign in the neighborhood of $\bar{w}$, that is to say,

$$
\exists h>0, a_{1} \in(\bar{w}-h, \bar{w}), a_{2} \in(\bar{w}, \bar{w}+h)
$$

$$
\text { such that } g\left(a_{1}\right) g\left(a_{2}\right)<0 \text {. }
$$

On the one hand, assume now that

$$
\exists w_{1} \in(a, \bar{w}) \quad \text { such that } g\left(w_{1}\right)<0 .
$$

However, by hypothesis (A.8),

$$
\exists w_{0} \in(a, \bar{w}) \quad \text { such that } g\left(w_{0}\right)>0 .
$$

Therefore, from (A.16) and (A.17), within the interval $\left(\min \left(w_{0}, w_{1}\right), \max \left(w_{0}, w_{1}\right)\right) \subseteq(a, \bar{w})$ a sign change occurs; so applying Bolzano's theorem, it follows that

$$
\exists \bar{w}_{1} \in(a, \bar{w}) \quad \text { such that } g\left(\bar{w}_{1}\right)=0,
$$

but this contradicts the hypothesis that the crossing point is unique (A.6). Thus $\nexists w_{1} \in(a, \bar{w})$ such that $g\left(w_{1}\right)<0$, that is,

$$
g(w)>0, \quad \forall w \in(a, \bar{w}),
$$

as we wanted to prove in (A.9).

On the other hand, notice that from (A.15) and (A.19) we have

$$
\exists h>0, a_{2} \in(\bar{w}, \bar{w}+h) \quad \text { such that } g\left(a_{2}\right)<0 .
$$

Assume now that

$$
\exists w_{2} \in(\bar{w}, b) \quad \text { such that } g\left(w_{2}\right)>0 .
$$

Therefore, from (A.20) and (A.21), within the interval $\left(\min \left(a_{2}, w_{2}\right), \max \left(a_{2}, w_{2}\right)\right) \subseteq(\bar{w}, b)$ a sign change occurs; so applying Bolzano's theorem, it follows that

$$
\exists \bar{w}_{2} \in(\bar{w}, b) \quad \text { such that } g\left(\bar{w}_{2}\right)=0,
$$

but this contradicts that the crossing point is unique (A.6). Thus $\nexists w_{2} \in(\bar{w}, b)$ such that $g\left(w_{2}\right)>0$, that is,

$$
g(w)<0, \quad \forall w \in(\bar{w}, b),
$$

as we wanted to prove in (A.10).

Lemma A.3. Let $f_{1}, f_{2}, \rho: \mathbb{R}^{+} \rightarrow \mathbb{R}$ be three real functions that satisfy the following.

(1) The following improper integrals of $f_{1}(x)$ and $f_{2}(x)$ are convergent and satisfy

$$
\int_{0}^{\infty} f_{2}(x) d x \leq \int_{0}^{\infty} f_{1}(x) d x
$$

(2) The $\rho(x)$ function is positive and monotonically decreasing for positive arguments

$$
\begin{gathered}
\rho(x)>0, \quad \forall x>0, \\
\rho^{\prime}(x)<0, \quad \forall x>0 .
\end{gathered}
$$

(3) There is an unique crossing point $\bar{x}$ for $f_{1}(x)$ and $f_{2}(x)$ such that

$$
\begin{aligned}
& f_{1}(x)>f_{2}(x), \quad \forall x \in(0, \bar{x}), \\
& f_{1}(x)<f_{2}(x), \quad \forall x \in(\bar{x}, \infty) .
\end{aligned}
$$


Proposition A.4. Then

$$
\int_{0}^{\infty} f_{1}(x) \rho(x) d x>\int_{0}^{\infty} f_{2}(x) \rho(x) d x .
$$

Proof. From (A.24), we have

$$
\int_{0}^{\infty}\left[f_{1}(x)-f_{2}(x)\right] d x \geq 0
$$

that is,

$$
\int_{0}^{\bar{x}}\left[f_{1}(x)-f_{2}(x)\right] d x \geq \int_{\bar{x}}^{\infty}\left[f_{2}(x)-f_{1}(x)\right] d x .
$$

Since $\rho(x)$ is a positive (A.25) and decreasing (A.26) function,

$$
\rho(x)>\rho(\bar{x})>0, \quad \forall x \in(0, \bar{x}),
$$

then, due to (A.27), we have

$$
\begin{array}{r}
{\left[f_{1}(x)-f_{2}(x)\right] \rho(x)>\left[f_{1}(x)-f_{2}(x)\right] \rho(\bar{x}),} \\
\forall x \in(0, \bar{x}) .
\end{array}
$$

According to [25, Theorem 3.3.4] we may integrate in (A.33) preserving the sense of the inequality:

$$
\begin{aligned}
& \int_{0}^{\bar{x}}\left[f_{1}(x)-f_{2}(x)\right] \rho(x) d x \\
& \quad>\rho(\bar{x}) \int_{0}^{\bar{x}}\left[f_{1}(x)-f_{2}(x)\right] d x .
\end{aligned}
$$

Similarly, we have

$$
\begin{aligned}
& 0<\rho(x)<\rho(\bar{x}), \quad \forall x \in(\bar{x}, \infty), \\
& \begin{aligned}
{\left[f_{2}(x)-f_{1}(x)\right] \rho(x)<\left[f_{2}(x)-f_{1}(x)\right] \rho(\bar{x}), } \\
\forall x \in(\bar{x}, \infty) ;
\end{aligned}
\end{aligned}
$$

thus

$$
\begin{aligned}
\int_{\bar{x}}^{\infty} & {\left[f_{2}(x)-f_{1}(x)\right] \rho(x) d x } \\
& <\rho(\bar{x}) \int_{\bar{x}}^{\infty}\left[f_{2}(x)-f_{1}(x)\right] d x \\
& \leq \rho(\bar{x}) \int_{0}^{\bar{x}}\left[f_{1}(x)-f_{2}(x)\right] d x \\
& <\int_{0}^{\bar{x}}\left[f_{1}(x)-f_{2}(x)\right] \rho(x) d x,
\end{aligned}
$$

where we have taken into account (A.31) and (A.34). Rewrite now (A.36) as

$$
\int_{0}^{\infty}\left[f_{1}(x)-f_{2}(x)\right] \rho(x) d x>0,
$$

which is just (A.29), as we wanted to prove.

\section{Conflict of Interests}

The authors declare that there is no conflict of interests regarding the publication of this paper.

\section{References}

[1] S. Malkin, Grinding Technology: Theory and Application of Machining with Abrasives, Ellis Horwood, John Wiley \& Sons, 1989.

[2] J. B. J. W. Hegeman, J. T. M. de Hosson, and G. de With, "Grinding of WC-Co hardmetals," Wear, vol. 248, no. 1-2, pp. 187-196, 2001.

[3] S. Malkin and C. Guo, "Thermal analysis of grinding," CIRP Annals-Manufacturing Technology, vol. 56, no. 2, pp. 760-782, 2007.

[4] C. Guo and S. Malkin, "Analysis of energy partition in grinding," Journal of Engineering for Industry, vol. 117, no. 1, pp. 55-61, 1995.

[5] S. Malkin and R. B. Anderson, "Thermal aspects of grinding: part 1-energy partition," Journal of Engineering for Industry, vol. 96, no. 4, pp. 1177-1183, 1974.

[6] A. S. Lavine and B. F. von Turkovich, "Thermal aspects of grinding: the effects of heat generation at the shear planes," CIRP Annals-Manufacturing Technology, vol. 40, no. 1, pp. 343-345, 1991.

[7] A. S. Lavine, "An exact solution for surface temperature in down grinding," International Journal of Heat and Mass Transfer, vol. 43, no. 24, pp. 4447-4456, 2000.

[8] M. N. Morgan, A. R. Jackson, H. Wu, V. Baines-Jones, A. Batako, and W. B. Rowe, "Optimisation of fluid application in grinding," CIRP Annals-Manufacturing Technology, vol. 57, no. 1, pp. 363366, 2008.

[9] J. C. Jaeger, "Moving sources of heat and the temperature at sliding contracts," Proceedings of the Royal Society of New South Wales, vol. 76, pp. 204-224, 1942.

[10] N. R. DesRuisseaux, Thermal aspects of grinding processes [Ph.D. dissertation], University of Cincinnati, 1968.

[11] W. J. Sauer, Thermal aspects of grinding [Ph.D. thesis], CarnegieMellon University, 1971.

[12] H. S. Carslaw and J. C. Jaeger, Conduction of Heat in Solids, Oxford Science Publications, Oxford, UK, 1947.

[13] N. R. DesRuisseaux and R. D. Zerkle, "Temperature in semiinfinite and cylindrical bodies subjected to moving heat surfaces and surface Cooling," Journal of Heat Transfer, vol. 92, pp. 456-464, 1970.

[14] S. Ohishi and Y. Furukawa, "Analysis of workpiece temperature and grinding burn in creep feed grinding," Bulletin of the JSME, vol. 28, no. 242, pp. 1775-1781, 1985.

[15] I. Zarudi and L. C. Zhang, "A revisit to some wheel-workpiece interaction problems in surface grinding," International Journal of Machine Tools and Manufacture, vol. 42, no. 8, pp. 905-913, 2002.

[16] J. L. González-Santander, J. M. Isidro, and G. Martín, "An analysis of the transient regime temperature field in wet grinding," Journal of Engineering Mathematics, vol. 90, pp. 141-171, 2015.

[17] D. L. Skuratov, Y. L. Ratis, I. A. Selezneva, J. Pérez, P. Fernández de Córdoba, and J. F. Urchueguía, "Mathematical modelling and analytical solution for workpiece temperature in grinding," Applied Mathematical Modelling, vol. 31, no. 6, pp. 1039-1047, 2007. 
[18] J. L. González-Santander and G. Martín, “Closed form expression for the surface temperature in wet grinding: application to maximum temperature evaluation," Journal of Engineering Mathematics, vol. 90, pp. 173-193, 2015.

[19] M. Mahdi and L. Zhang, "The finite element thermal analysis of grinding processes by ADINA," Computers and Structures, vol. 56, no. 2-3, pp. 313-320, 1995.

[20] N. N. Lebedev, Special Functions and their applications, Dover Publications, New York, NY, USA, 1972.

[21] F. W. J. Olver, D. W. Lozier, R. F. Boisvert, and C. W. Clark, NIST Handbook of Mathematical Functions, Cambridge University Press, New York, NY, USA, 2010.

[22] J. B. Conway, Functions of One Complex Variable, vol. 11 of Graduate Texts in Mathematics, Springer, Berlin, Germany, 2nd edition, 1978.

[23] D. S. Mitrinović, Analytic Inequalities, Springer, New York, NY, USA, 1970.

[24] T. M. Apostol, Calculus, vol. 1, John Wiley \& Sons, New York, NY, USA, 1967.

[25] W. F. Trench, Introduction to Real Analysis, Books and Monographs, Book 7, 2013, http://digitalcommons.trinity.edu/ mono/7.

[26] I. S. Gradsthteyn and M. Ryzhik, Table of Integrals, Series and Products, Academic Press, New York, NY, USA, 7th edition, 2007.

[27] V. I. Murav'ev, A. V. Yakimov, and A. V. Chernyshev, "Effect of deformation, welding, and electrocontact heating on the properties of titanium alloy VT20 in pressed and welded structures," Metal Science and Heat Treatment, vol. 45, no. 11-12, pp. 419-422, 2003. 


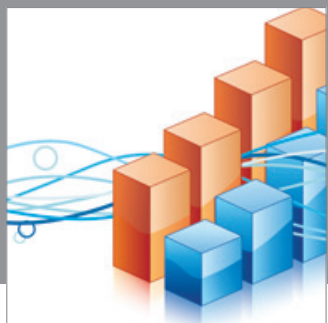

Advances in

Operations Research

mansans

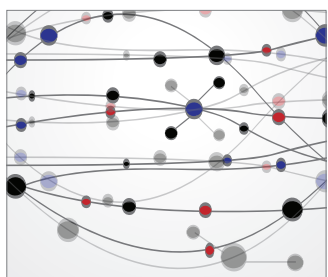

The Scientific World Journal
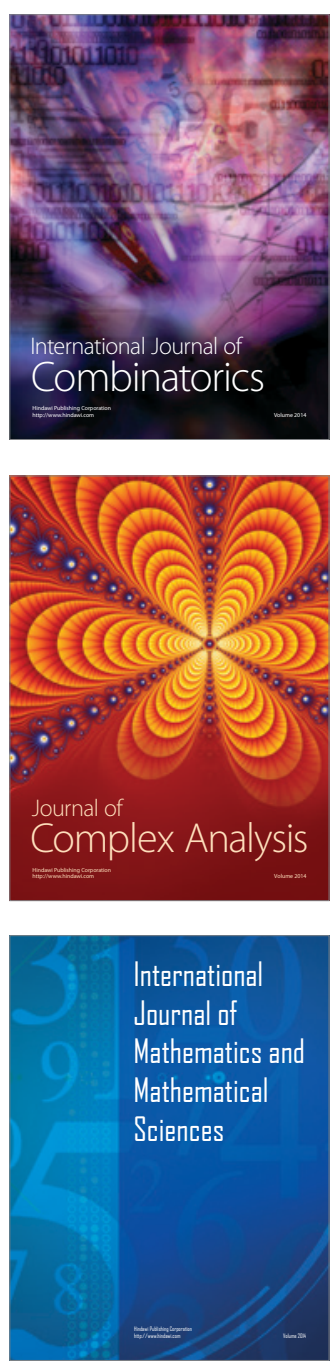
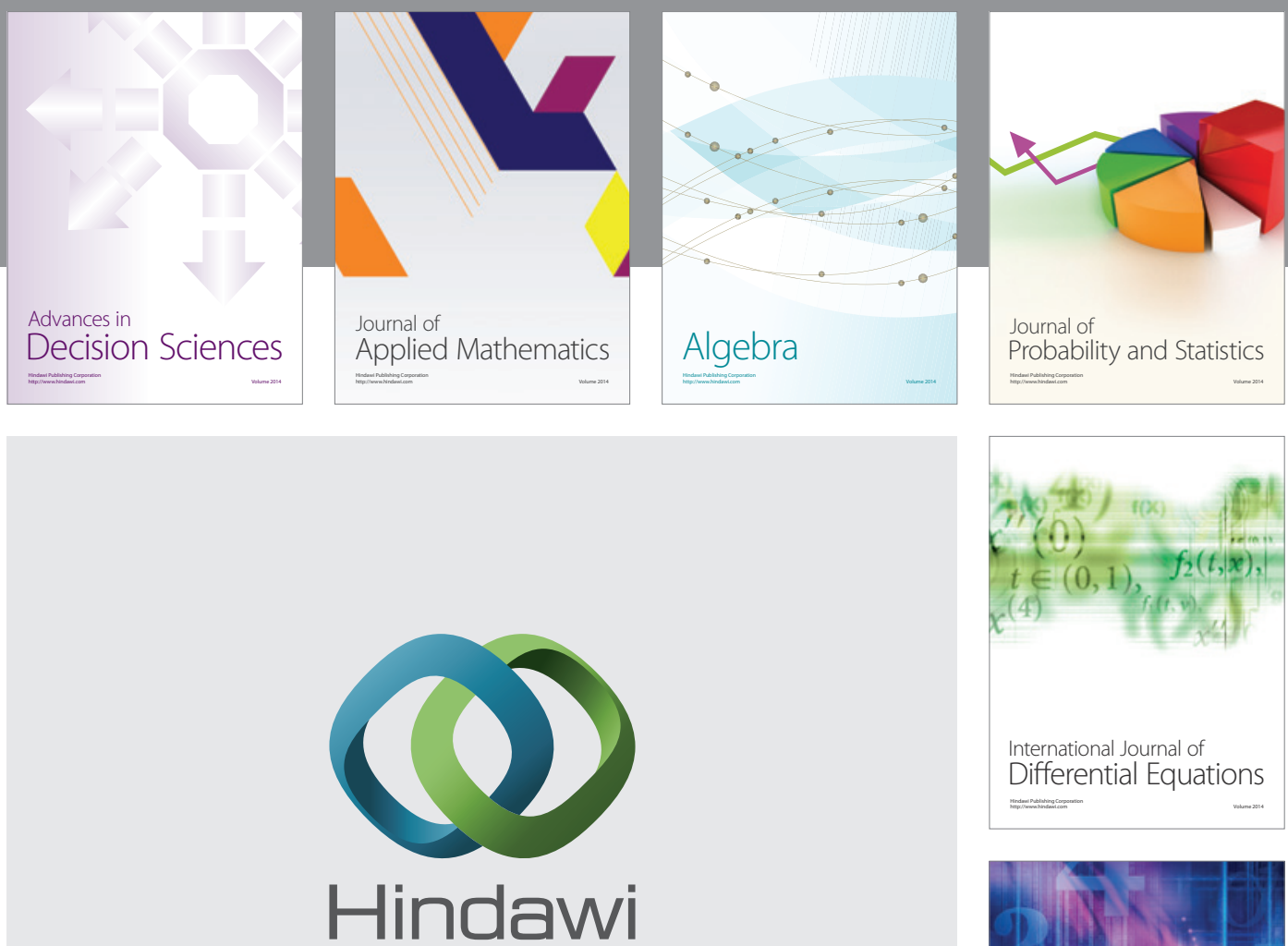

Submit your manuscripts at http://www.hindawi.com
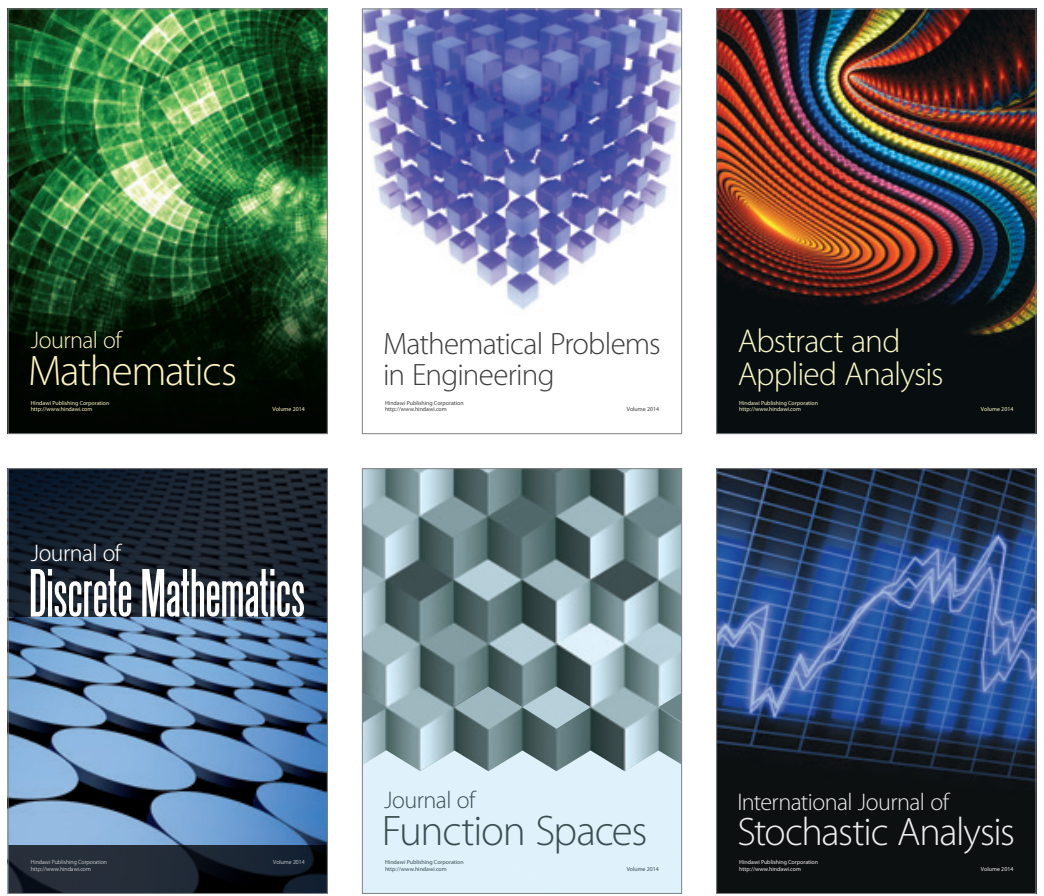

Journal of

Function Spaces

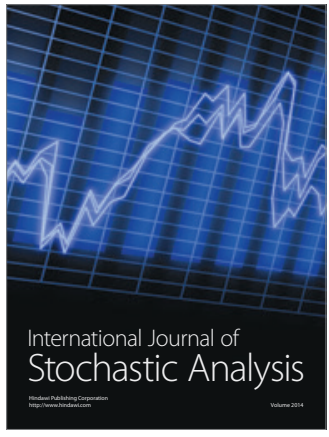

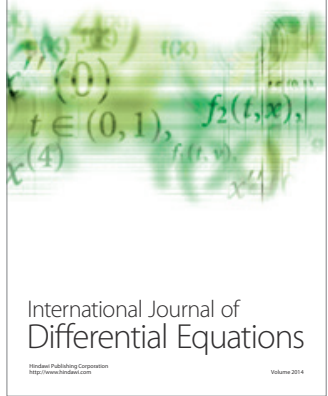
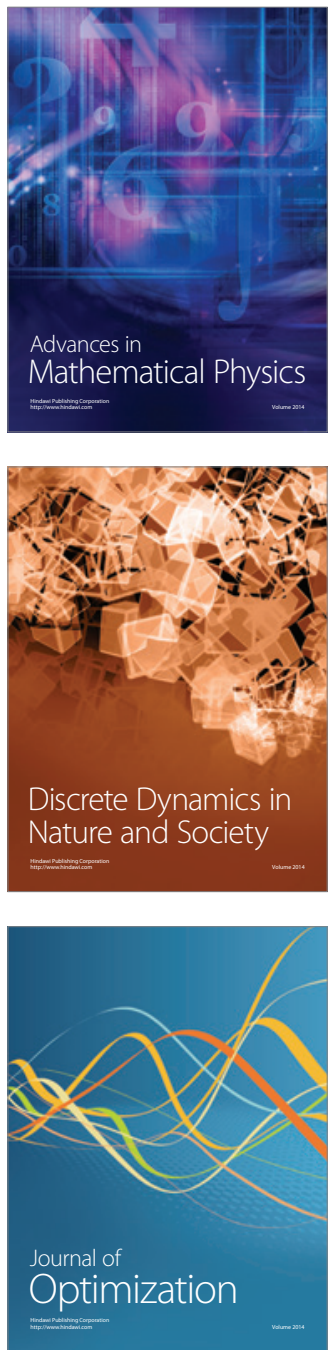